\title{
Inhibition of CCR7/CCL19 Axis in Lesional Skin Is a Critical Event for Clinical Remission Induced by TNF Blockade in Patients with Psoriasis
}

\author{
Francesca Bosè, ${ }^{*}$ Luciana Petti, ${ }^{*}$ Marco Diani, ${ }^{\dagger}$ Claudia Moscheni, ${ }^{\ddagger}$ Silvia Molteni, ${ }^{*}$ Andrea Altomare, ${ }^{\dagger}$ Riccardo L. Rossi, \\ Daniela Talarico, "Raffaella Fontana," Vincenzo Russo," Gianfranco Altomare, ${ }^{\dagger}$ and Eva Reali*§
}

\begin{abstract}
From the Laboratory of Translational Immunology,* Istituto di Ricovero e Cura a Carattere Scientifico (IRCCS) Istituto Ortopedico Galeazzi, Milan; the Department of Dermatology, ${ }^{\dagger}$ IRCCS Istituto Ortopedico Galeazzi and University of Milan, Milan; the Department of Biomedical and Clinical Sciences Luigi Sacco, ${ }^{\ddagger}$ University of Milan, Milan; the Department of Genomics and Molecular Biology, ${ }^{\S}$ National Institute of Molecular Genetics (INGM), Milan; and the Division of Genetics and Cell Biology" and the Cancer Gene Therapy Unit, ${ }^{\|}$IRCCS Ospedale San Raffaele, Milan, Italy
\end{abstract}

Accepted for publication April 3, 2013.

Address correspondence to Eva Reali, Ph.D, Laboratory of Translational Immunology, IRCCS Istituto Ortopedico Galeazzi, via Riccardo Galeazzi 4, 20161 Milan, Italy. E-mail: eva.reali@grupposandonato.it.

\begin{abstract}
Despite the evidence that tumor necrosis factor (TNF) inhibitors block TNF and the downstream inflammatory cascade, their primary mechanism of action in inhibiting the self-sustaining pathogenic cycle in psoriasis is not completely understood. This study has the aim to identify early critical events for the resolution of inflammation in skin lesions using anti-TNF therapy. We used a translational approach that correlates gene expression fold change in lesional skin with the Psoriasis Area and Severity Index score decrease induced by TNF blockade after 4 weeks of treatment. Data were validated by immunofluorescence microscopy on skin biopsy specimens. We found that the anti-TNF-modulated genes that mostly associated with the clinical amelioration were $\mathrm{Ccr} 7$, its ligand, $\mathrm{Ccl} 19$, and dendritic cell maturation genes. Decreased expression of T-cell activation genes and Vegf also associated with the clinical response. More important, the down-regulation of C $\mathrm{Cr} 7$ observed at 4 weeks significantly correlated with the clinical remission occurring at later time points. Immunofluorescence microscopy on skin biopsy specimens showed that reduction of $C \mathrm{CR} 7^{+}$cells and chemokine ligand (CCL) 19 was paralleled by disaggregation of the dermal lymphoid-like tissue. These data show that an early critical event for the clinical remission of psoriasis in response to TNF inhibitors is the inhibition of the CCR7/ CCL19 axis and support its role in psoriasis pathogenesis. (Am J Pathol 2013, 183: 413-421; http:// dx.doi.org/10.1016/j.ajpath.2013.04.021)
\end{abstract}

In the past years, several studies have contributed to the understanding of important immunopathogenic mechanisms of psoriasis, evidencing that psoriatic lesions evolve from the cross talk between $\mathrm{T}$ cells, dendritic cells (DCs), skin epithelium, and connective tissue to produce a complex interplay that perpetuates the inflammatory process. ${ }^{1,2}$

The primary role of T-cell and DC interaction in sustaining skin inflammation has been demonstrated by immunofluorescence microscopy on psoriatic skin lesions and by ex vivo experiments. Evidence has also been provided by functional genomic studies, showing the higher expression of DC genes, $\mathrm{T}$-cell activation genes, and genes encoding chemokines (Il8 and $\mathrm{Ccl19}$ ) in psoriatic skin lesions compared with normal control skin. ${ }^{1-5}$
Gene expression and microscopy studies on skin samples from patients treated with tumor necrosis factor (TNF) blocking agents have supported the role of myeloid DCs in the pathogenesis of the disease and in the generation of T-helper cell (Th) 1 and Th17 responses. Indeed, TNF inhibitors have been shown to down-regulate the expression of IL-23 and Th17 cytokines and the infiltration of myeloid CD11 $\mathrm{c}^{+}$cells and $\mathrm{T}$ cells in psoriatic plaques. ${ }^{6,7}$

In addition to the effective treatment of psoriasis, TNF inhibitors are highly effective in the treatment of other autoimmune and inflammatory diseases, such as rheumatoid arthritis and inflammatory bowel diseases. ${ }^{8}$

Supported by a Pfizer Italia unrestricted educational grant. 
In target tissues, TNF inhibitors modulate, in a similar manner, the expression of a wide panel of genes involved in immune and inflammatory responses. A few of these genes represent direct targets of TNF. These include early inflammatory genes, chemokine genes (Il8, Illb, Cxcl10, and Cxcl11), and the gene encoding vascular endothelial growth factor (VEGF). ${ }^{9-11}$ Other genes are modulated by indirect mechanisms and reflect the changes in the cell and cytokine network occurring within the target tissues. ${ }^{6,7,12,13}$

Emerging evidence indicates that, in different pathological conditions, the disease reversal is likely to be mainly dependent on the modulation of the pathways that mostly contribute to disease pathogenesis. ${ }^{13-15}$

In patients with psoriasis, the down-modulation of IL-17 pathway genes was shown to be associated with the clinical response after 12 weeks of etanercept treatment, whereas the down-modulation of early inflammatory genes that are direct targets of TNF was not associated with the clinical response. ${ }^{7}$ Despite this evidence, the primary mechanisms by which TNF inhibitors directly interrupt the self-maintaining pathogenic cycle in psoriasis remain to be established.

The current study had the aim to determine the key mechanisms that initiate the resolution of inflammation in psoriatic skin lesions on TNF blockade, and we found that the inhibition of the CCR7/chemokine ligand (CCL) 19 axis and the down-regulation of DC maturation genes highly correlate with the clinical remission and the disaggregation of the lymphoid-like tissue in the upper dermis.

\section{Materials and Methods}

\section{Patient Population}

Nine patients from the Department of Dermatology, Istituto di Ricovero e Cura a Carattere Scientifico Istituto Ortopedico Galeazzi (Milan, Italy), with a diagnosis of cutaneous psoriasis with a Psoriasis Area and Severity Index (PASI) $>8$, all ranging in age from 26 to 81 years, were enrolled in the study. Criteria for patient selection included the absence of comorbidities, such as diabetes mellitus, genetic diseases, and acute and chronic systemic or cutaneous infections during sample collection; all patients were negative at the screening for hepatitis $\mathrm{B}$ virus, hepatitis $\mathrm{C}$ virus, HIV, and tuberculosis infections. Patients undergoing treatment with cyclospotin $\mathrm{A}$, methotrexate, or systemic corticosteroids during anti-TNF therapy or within 3 weeks before the beginning of the anti-TNF therapy were excluded from the study. Patients were treated with $25 \mathrm{mg}$ s.c., twice a week, TNF-blocking agents etanercept, $5 \mathrm{mg} / \mathrm{kg}$ i.v. at weeks 0,2 , and 6 , and every 8 weeks infliximab, or $80 \mathrm{mg}$ s.c., twice in the first infusion, $40 \mathrm{mg}$ after 1 week, and then $40 \mathrm{mg}$ every 15 days adalimumab. Full-thickness punch biopsy specimens were collected from lesional psoriasis skin of individual patients under untreated conditions (baseline) and after 4 weeks of therapy in the same lesional area.

The study was approved by the local ethical commission, and written informed consent was obtained from all patients before they entered the study, which was performed in accordance with the Declaration of Helsinki. The patient's demographic and clinical characteristics at baseline and during therapy are summarized in Table 1 .

\section{Clinical Evaluation of Disease Severity and Response to the Therapy}

The clinical severity of psoriasis and the response to the therapy were evaluated calculating the PASI score for each patient at baseline and during therapy at 4 and 12 weeks.

\section{RNA Extraction and qPCR}

Skin biopsy specimens from patients with psoriasis were collected at baseline and after 4 weeks of anti-TNF therapy and frozen in liquid nitrogen. Total RNA was extracted using the RNeasy fibrous tissue Mini Kit (Qiagen, Milan) under RNase-free conditions, according to the instructions of the manufacturer. RNA quantity and quality were assessed using the Nanodrop ND-100 spectrophotometer (Nanodrop Technologies, Milan) and the 2100 Bioanalyzer (Agilent RNA 6000 nano kit; Agilent Technologies, Inc., Milan), with a 260/ 280 ratio $\geq 1.5$ and an RNA integrity number $\geq 7$ for most samples. An equal amount of RNA (500/1000 ng per sample) from paired biopsy specimens (baseline and 4 weeks' anti-TNF therapy) was retrotranscribed in cDNA by a HighCapacity cDNA Reverse Transcription Kit (Applied Biosystems, Monza, Italy), as described by the manufacturer. Real-time quantitative PCR (qPCR) was performed by a TaqMan Low Density Array Gene Signature Panel (Human Immune Panel; Applied Biosystems), which allows simultaneous evaluation of the expression of 96 genes in duplicate. qPCR was performed using a 7900 HT Real Time PCR System (Applied Biosystems) for human gene profiling. Relative gene expression was quantified using a $\Delta \mathrm{C}_{\mathrm{T}}$ method, normalizing data to the mean of $\beta$-actin/18S/glyceraldehyde3 -phosphate dehydrogenase as housekeeping genes.

\section{H\&E Staining and Immunofluorescence}

Skin biopsy specimens were fixed overnight in PBS-buffered $10 \%$ paraformaldehyde at $4^{\circ} \mathrm{C}$, embedded in optimal cutting temperature compound, and frozen at $-80^{\circ} \mathrm{C}$. Cryosections were stained with $\mathrm{H} \& \mathrm{E}$ (Bio Optica, Milan), according to standard procedures. For immunofluorescence microscopy, cryosections were treated for antigen retrieval and blocking, according to the standard procedure, ${ }^{16}$ and then stained by incubation overnight at $4^{\circ} \mathrm{C}$ with the following primary antibodies in blocking solution: monoclonal mouse anti-human CCR7 (1:50; MAB197, clone 150503; R\&D Systems, Minneapolis, MN), polyclonal goat anti-human macrophage inflammatory protein $3 \beta$ (CCL19; 1:50; ab10389; Abcam, Cambridge, UK), monoclonal rabbit anti-human CD11c/ ITGAX (1:100; 1975-1; Epitomics, Burlingame, CA), polyclonal goat anti-human CD4 (1:50; AF-379-NA; R\&D 
Table 1 Characteristics of Patients Included in the Study

\begin{tabular}{|c|c|c|c|c|c|c|c|c|c|}
\hline \multicolumn{3}{|c|}{ Patients with psoriasis } & \multirow[b]{2}{*}{ Disease } & \multirow[b]{2}{*}{ Anti-TNF therapy } & \multicolumn{3}{|l|}{ PASI } & \multicolumn{2}{|c|}{$\%$ PASI decrease } \\
\hline No. & Sex & Age & & & Baseline & 1 month & 3 months & 1 month & 3 months \\
\hline 1 & M & 67 & Pso & Adalimumab & 18.3 & 2.7 & 2.7 & 85.25 & 85.25 \\
\hline 3 & M & 26 & Pso & Infliximab & 16 & 4 & 2 & 75 & 87.5 \\
\hline 4 & $\mathrm{~F}$ & 71 & Pso & Etanercept & 12 & 7.5 & 3.5 & 37.5 & 70.83 \\
\hline 5 & M & 60 & PsA & Etanercept & 21.5 & 14.2 & 8.5 & 33.95 & 60.47 \\
\hline 8 & M & 60 & Pso & Etanercept & 19.8 & 14.8 & 11 & 25.25 & 44.44 \\
\hline 9 & M & 41 & PsA & Adalimumab & 25 & 20.3 & 20.3 & 18.8 & 18.8 \\
\hline
\end{tabular}

F, female; M, male; PASI, Psoriasis Area and Severity Index; Pso, psoriasis; PsA, psoriasis and psoriatic arthritis.

Systems), polyclonal rabbit anti-human CD3 (1:100; CP 215; Biocare Medical, Concord, CA), and monoclonal mouse anti-human CD19 (1:100; CM 310; Biocare Medical). Then, slides were incubated with fluorescent secondary goat anti-mouse Alexa488, rabbit anti-goat Alexa647, goat anti-rabbit Alexa647, or goat anti-rabbit Alexa488, in blocking solution (final dilution, 1:600; Invitrogen, Monza) for 1 hour at room temperature. At the end, nuclei were stained with Hoechst 33258, and sections were washed in PBS, mounted with a specific medium (DakoCytomation, Milan), and examined by using a confocal laser-scanning microscope (TCS SP5; Leica Microsystem Microscope, Milan).

Control sections were processed under identical conditions using mouse, goat, or rabbit serum instead of the primary antibodies (Supplemental Figure S1).

Signal quantification was performed using ImageJ version $1.47 \mathrm{~g}(\mathrm{NIH}$, Bethesda, $\mathrm{MD})$ and refers to the area of the fluorescence signal $\left(\mu \mathrm{m}^{2}\right)$. Briefly, the fluorescence signal was quantified, within each field, normalizing the red or green area to the blue area of cell nuclei. At least 10 fields for each staining were quantified. Significance levels were calculated by Student's $t$-test for unpaired data (Prism version 5 Software Inc.; La Jolla, CA).

\section{Statistical Analysis and Differential Expression Criteria}

$\mathrm{MeV}$ version 4.6 (TM4 microarray software suite, Boston, MA), was used for gene expression data analysis.

Differentially expressed genes between baseline and 4 weeks' treatment with TNF inhibitors in individual patients were calculated by mRNA fold change (expressed as $\log _{2} 4$ weeks/baseline gene expression level).

The correlation between changes in gene expression within the psoriatic plaque and clinical amelioration after anti-TNF therapy (4 or 12 weeks), expressed as percentage of PASI score reduction, was analyzed by Spearman's rank test (indicated as Spearman's $r$ ) (Figure 1).

Pearson's correlation was used to correlate the gene expression fold change of two genes. For all tests, the level of statistical significance was set at $P<0.05$.

\section{Generation of DCs}

A total of $5 \times 10^{7}$ to $6 \times 10^{7}$ peripheral blood mononuclear cells from healthy donors were allowed to adhere for 1 hour at $37^{\circ} \mathrm{C}$. Adherent cells were then cultured in RPMI 1640 medium $10 \%$ fetal bovine serum supplemented with 1000 $\mathrm{U} / \mathrm{mL}$ granulocyte-macrophage colony-stimulating factor (Schering-Plough Co-Innishannon, County Cork, Ireland), $500 \mathrm{U} / \mathrm{mL}$ IL-4 (ImmunoTools, Friesoythe, Germany), and 50 $\mathrm{mmol} / \mathrm{L} \beta$-mercaptoethanol. At day 5, $8 \times 10^{5}$ differentiated DCs were stimulated with $10 \mathrm{ng} / \mathrm{mL} \mathrm{TNF} \alpha$ and $10 \mathrm{ng} / \mathrm{mL}$ IL-1 $\beta$ in the presence or absence of $1 \mu \mathrm{g} / \mathrm{mL}$ etanercept for 48 hours. At day 7, mature DCs were analyzed by fluorescence-activated cell sorting (FACS) for the expression of CD83 and CCR7 markers. Anti-CD83 monoclonal antibodies were from BD Biosciences (San Jose, CA), and antiCCR7 was from MBL International Corp (Woburn, MA). Isotype controls from the same manufacturers were used. Samples were run by an FACSCanto flow cytometer (BD Biosciences, Monza, Italy) and analyzed by FlowJo software version 6.4.7 (Ashland, OR) by gating on live cells.

For qPCR analysis on DCs, cells were cultured for 24 hours under the same conditions indicated before; RNA was extracted using a mirVana miRNA Isolation kit (Ambion, Monza), retrotranscribed in cDNA by a High-Capacity cDNA Reverse Transcription Kit (Applied Biosystems), as described by the manufacturer, and analyzed by CCR7 and CCL19 TaqMan gene expression assays (commercially available single assay; Applied Biosystems) and a 7900 HT Real Time PCR System (Applied Biosystems) for human gene profiling. Relative gene expression was quantified using the $\Delta \mathrm{C}_{\mathrm{T}}$ method, normalizing data to the mean of $\beta$-actin/18S/glyceraldehyde-3-phosphate dehydrogenase as housekeeping genes.

\section{Statistical Analysis}

For FACS analysis of CD83 and CCR7 expression on mature DCs and qPCR analysis of $\mathrm{Ccr} 7$ and $C c l 19$ expression on DCs, significance levels were calculated by a one-tailed paired Student's $t$-test (Prism version 5 Software Inc.). 
A

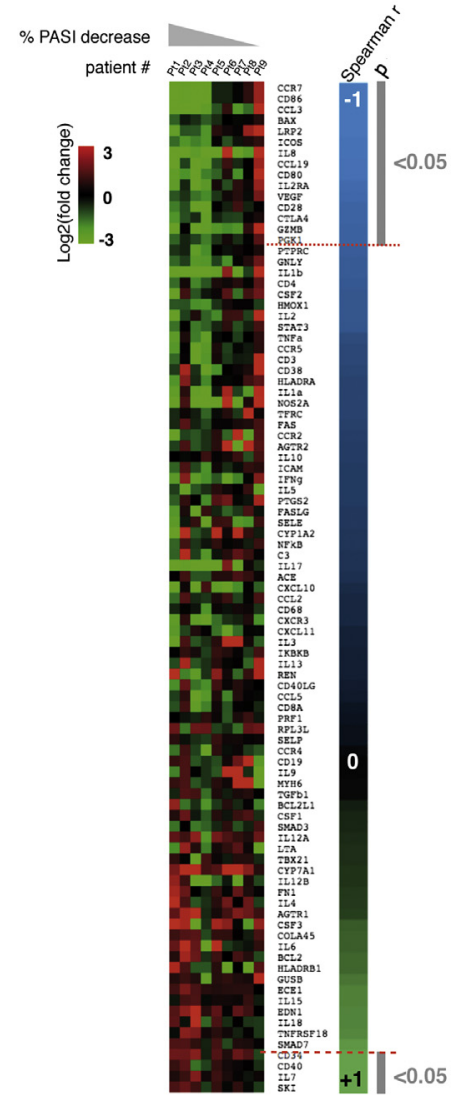

B

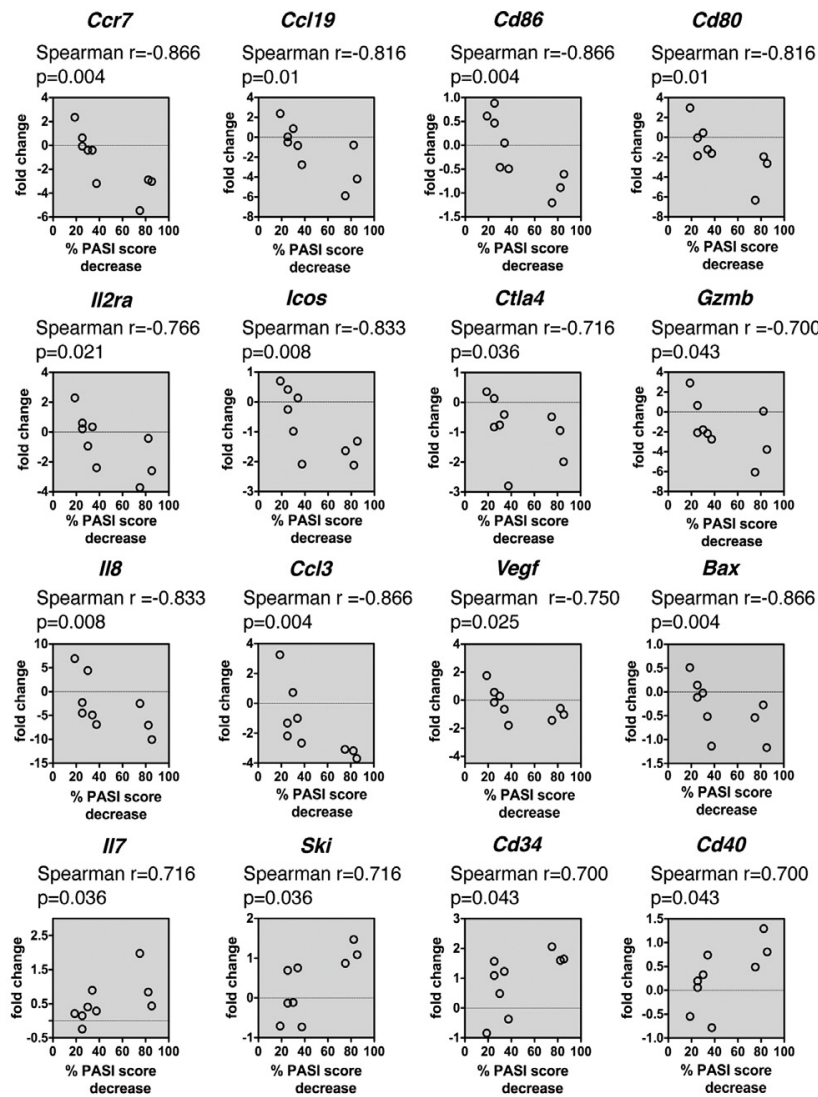

Figure 1 Clinical remission correlates with reduction of $C \mathrm{cr} 7, \mathrm{C}(19$, and DC-associated gene expression within the psoriatic plaque. Gene expression analysis was performed by TaqMan Low Density Array (Applied Biosystems) on skin biopsy specimens collected from nine patients with psoriasis at baseline and after 4 weeks of treatment with TNF inhibitors. A: Heat map of gene expression modulation induced by anti-TNF therapy (calculated by log ${ }_{2}$ fold change 4 weeks/baseline gene expression level). Patients are ordered by percentage PASI decrease and ranked according to Spearman's correlation between fold change and clinical response. Spearman's $r$ is depicted by the vertical gradient bar, and the significance level is shown (gray bar, $P<0.05)$. B: Correlations for single genes are expressed as Spearman's $r$, and significance level is calculated by Student's paired $t$-test (gaussian approximation).

\section{Results}

Decreased Expression of Ccr7, Ccl19, and DC Maturation Genes in Psoriatic Skin Lesions Highly Correlates with Clinical Remission in Response to Anti-TNF Therapy

Gene expression modulation induced by anti-TNF therapy in psoriatic lesions was evaluated by qPCR on skin biopsy specimens collected from patients at baseline and after 4 weeks of treatment. We used a TaqMan Low Density Array Gene Signature Human Immune Panel, which allows the simultaneous evaluation of 96 genes involved in the immune and inflammatory response.

To identify the genes putatively involved in the early phases of the resolution of inflammation in psoriatic plaques induced by TNF inhibitors, we first ordered the samples according to a decreasing PASI pattern, with patient 1 having the highest decrease and patient 9 having the lowest decrease (Table 1). We then evaluated, for each gene, the correlation between the changes of expression and the clinical remission after 4 weeks of treatment in individual patients. To this purpose, we performed a Spearman's rank analysis to correlate the mRNA fold change (expressed as $\log _{2} 4$ weeks/ baseline gene expression level) with the percentage of PASI score reduction for each patient. A heat map representing gene expression modulation induced by 4 weeks of treatment was generated by ordering the gene list according to the correlation analysis results, from the highest negative correlation (Spearman's $r=-1$ ) to the highest positive correlation (Spearman's $r=1$ ), as shown in Figure 1A. In the upper part of the heat map are shown 15 genes that have a significant $(P<0.05)$ negative fold-change/response correlation (Figure 1A), and in the lower part, the four genes having a positive fold-change/response correlation (Figure 1A). Figure 1B shows the correlation between gene expression fold change after 4 weeks of treatment and the percentage of PASI score decrease for each patient for genes with $P<0.05$. Among genes that showed a negative correlation, those that mostly correlated with the clinical response were the chemokine receptor gene, $C c r 7$ (Spearman's $r=-0.866, P=$ $0.004)$, characteristically expressed by maturing DCs and by central memory/naïve T cells, and the DC maturation genes, $C d 86$ (Spearman's $r=-0.866, P=0.004$ ) and $C d 80$ 
Pearson $r=0.917$ $\mathrm{p}=0.0005$

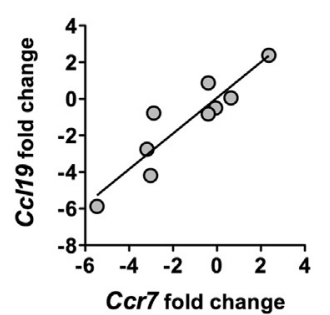

Pearson $r=0.901$ $\mathrm{p}=0.0009$

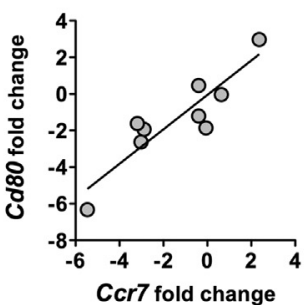

Pearson $r=0.873$ $\mathrm{p}=0.002$

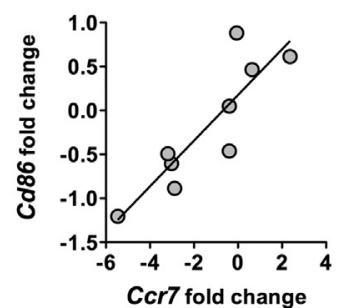

Pearson $\mathrm{r}=0.868$ $\mathrm{p}=0.002$

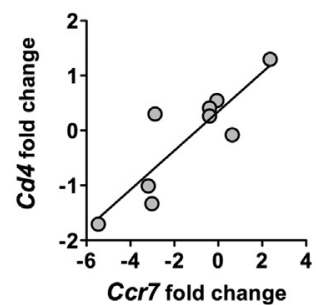

Figure 2 Decreased expression of Ccr7 correlates with the reduction of $C c 119, C d 80, C d 86$, and $C d 4$ expression. Correlation between Ccr7 and Ccl19, Cd80, Cd86, or $C d 4$ down-regulated expression (calculated as $\log _{2}$ fold change 4 weeks/baseline gene expression level) was evaluated by Pearson's analysis. Pearson's correlation coefficient, expressed as Pearson's $r$ value, and significance level, calculated by Student's $t$-test, are indicated.

(Spearman's $r=-0.816, P=0.01)$. Notably, decreased expression of the gene encoding the CCR7 ligand, Ccll9, was also strongly correlated with the clinical response (Spearman's $r=-0.816, P=0.01$ ) (Figure 1B).

We also found that reduction of the expression of genes associated with T-cell activation, Il2ra (Spearman's $r=$ $-0.766, P=0.021$ ), Icos (Spearman's $r=-0.833, P=$ 0.008 ), and Ctla4 (Spearman's $r=-0.716, P=0.036$ ), was highly correlated with the clinical response to TNF inhibitors (Figure 1B). In addition, a correlation was observed between the down-regulation of $G z m b$, encoding the granzyme B cytotoxic enzyme, and the clinical response to the treatment (Spearman's $r=-0.700, P=0.043$ ) (Figure 1B). The lack of correlation of $C d 4$ and $C d 8$ expression with disease amelioration suggests that $\mathrm{T}$-cell activation, rather than infiltration, is involved in the clinical response at an early time point during therapy (Supplemental Table S1).

Conceivably, we found a significant correlation between the down-regulation of known TNF-target genes, such as $I l 8$ (Spearman's $r=-0.833, P=0.008$ ) and $C c l 3$ (Spearman's $r=-0.866, P=0.004)$, and the pro-apoptotic gene, $B a x$ (Spearman's $r=-0.866, P=0.004$ ), and the clinical response to TNF inhibitors. More important, we also found a correlation between the decreased expression of the proangiogenic TNF-target gene, Vegf, and the decrease of PASI score (Spearman's $r=-0.750, P=0.025$ ) (Figure 1B). The complete set of genes, together with Spearman's $r$ values and significance levels, are reported in Supplemental Table S1.

Together, these data evidenced that resolution of psoriatic lesions in response to TNF-blocking treatment is strongly associated with the early inhibition of the CCR7/CCL19 axis and with the reduction of DC maturation genes, Vegf, and T-cell activation genes.

We next calculated the correlation between the fold change expression of $\mathrm{Ccr} 7$ and its ligand, $\mathrm{Ccl} 19$, and the expression of DC maturation genes and T-cell lineage marker, CD4. As shown in Figure 2, Pearson's analysis evidenced a high correlation between the down-regulation of $\mathrm{Ccr} 7$ and the decrease of $\mathrm{Ccl} 19$ expression induced by TNF blockade (Pearson's $r=0.917, P=0.0005$ ), underlining the stringent comodulation of these genes in individual patients in response to TNF blockade.
In addition, a strong correlation between $\mathrm{Ccr} 7$ fold change and the fold change of the DC maturation genes, $C d 80$ and $C d 86$ [Pearson's $r=0.901(P=0.0009)$ and Pearson's $r=$ $0.873(P=0.002)$, respectively], was also observed. Decreased expression of $\mathrm{Ccr} 7$ also correlated with the down-regulation of $C d 4$ expression (Pearson's $r=0.868, P=0.002$ ) and $C d 3$ expression (Pearson's $r=0.870, P=0.002$ ), whereas it did not correlate with $C d 8$ expression fold change (Pearson's $r=0.555, P=0.12$ ), thus suggesting that mainly CD4 T cells expressing CCR7 (central memory or naive) were reduced in parallel with the inhibition of the CCR7/CCL19 axis.

Furthermore, we found that the expression of four genes ( $C d 40, C d 34, I l 7$, and $S k i)$ was positively correlated with PASI score decrease (Figure 1B). These up-regulated genes are likely to be associated with skin regeneration processes, in particular $C d 34$, a precursor cell marker, and $S k i$, which has recently emerged as a tissue-repair gene. ${ }^{17}$ Up-regulation of $C d 40$, expressed by immature antigen-capturing DCs, and Il7, mediating peripheral T-cell homeostasis, may reflect restored immunological homeostasis in the skin. ${ }^{18,19}$ Finally, to confirm the role of the selected down-regulated genes in inhibiting the pathogenic process in psoriatic skin, we evaluated the correlation with the clinical outcome at later time points. We performed a Spearman's rank analysis to evaluate the correlation between gene expression fold change at 4 weeks of treatment with TNF inhibitors (expressed as $\log _{2}$ 4 weeks/baseline gene expression level) and the clinical response after 12 weeks of treatment (expressed as percentage of PASI score decrease). The analysis evidenced that the early down-modulation of $\mathrm{Ccr} 7$, detected after 4 weeks, also correlated with the clinical remission occurring later on, at 12 weeks of treatment (Table 2). This strengthens the evidence that CCR7 down-regulation is a critical early event that evolves in disease resolution at later time points during therapy.

\section{Anti-TNF Therapy Reduces $\mathrm{CCR} 7{ }^{+} \mathrm{CD} 11 \mathrm{C}^{+} \mathrm{DCs}$ and $\mathrm{CCR}^{+}{ }^{+} \mathrm{CD}^{+}{ }^{+} \mathrm{T}$ Cells in the Dermis and Dissolved Lymphoid Aggregates}

To confirm the results obtained from gene expression analysis, we performed tissue microscopy experiments on full-thickness tissue sections from skin biopsy specimens 
Table 2 Correlation between Gene Expression Modulation by TNF Inhibitors and Clinical Response after 12 Weeks of Therapy

\begin{tabular}{|c|c|c|}
\hline Genes & Spearman's $r$ & $P$ value \\
\hline$C d 86$ & -0.950 & 0.0004 \\
\hline$I \cos$ & -0.850 & 0.006 \\
\hline Il2ra & -0.800 & 0.013 \\
\hline Ccr7 & -0.783 & 0.017 \\
\hline Ptprc & -0.778 & 0.017 \\
\hline$C d 28$ & -0.750 & 0.025 \\
\hline $\mathrm{Ccl3}$ & -0.716 & 0.036 \\
\hline Lrp2 & -0.683 & 0.050 \\
\hline $\mathrm{Cd} 80$ & -0.683 & 0.050 \\
\hline Gnly & -0.666 & 0.058 \\
\hline $\mathrm{Cd} 4$ & -0.650 & 0.066 \\
\hline Vegf & -0.616 & 0.085 \\
\hline Ccl19 & -0.616 & 0.085 \\
\hline Bax & -0.600 & 0.096 \\
\hline Il8 & -0.550 & 0.132 \\
\hline Ccr2 & -0.550 & 0.132 \\
\hline Ctla4 & -0.533 & 0.147 \\
\hline Il10 & -0.533 & 0.147 \\
\hline $\mathrm{Cd} 3$ & -0.516 & 0.161 \\
\hline Cor 5 & -0.516 & 0.161 \\
\hline Gzmb & -0.500 & 0.177 \\
\hline Hmox1 & -0.450 & 0.229 \\
\hline Ren & -0.443 & 0.229 \\
\hline Ifng & -0.433 & 0.249 \\
\hline Pgk1 & -0.400 & 0.291 \\
\hline Il5 & -0.400 & 0.291 \\
\hline Il2 & -0.383 & 0.312 \\
\hline Prf1 & -0.376 & 0.312 \\
\hline Stat3 & -0.366 & 0.336 \\
\hline$I l 1 b$ & -0.350 & 0.358 \\
\hline Faslg & -0.350 & 0.358 \\
\hline Csf2 & -0.300 & 0.436 \\
\hline Selp & -0.300 & 0.436 \\
\hline Il1a & -0.283 & 0.463 \\
\hline Сур1a2 & -0.283 & 0.463 \\
\hline Ace & -0.283 & 0.463 \\
\hline Hladra & -0.266 & 0.493 \\
\hline Fas & -0.266 & 0.493 \\
\hline Tnfa & -0.266 & 0.493 \\
\hline $\mathrm{Cd} 38$ & -0.250 & 0.520 \\
\hline Il13 & -0.233 & 0.551 \\
\hline Cxcl10 & -0.233 & 0.551 \\
\hline$N f k b$ & -0.216 & 0.580 \\
\hline Ccl5 & -0.216 & 0.580 \\
\hline Icam & -0.200 & 0.613 \\
\hline Cd68 & -0.183 & 0.643 \\
\hline Nos $2 a$ & -0.183 & 0.643 \\
\hline$C d 8$ & -0.183 & 0.643 \\
\hline Ptgs2 & -0.166 & 0.677 \\
\hline Rpl3l & -0.150 & 0.708 \\
\hline Tfrc & -0.116 & 0.775 \\
\hline$C 3$ & -0.116 & 0.775 \\
\hline Agtr2 & -0.116 & 0.775 \\
\hline$T b \times 21$ & -0.116 & 0.775 \\
\hline
\end{tabular}

(table continues)
Table 2 (continued)

\begin{tabular}{|c|c|c|}
\hline Genes & Spearman's $r$ & $P$ value \\
\hline Cxcl11 & -0.083 & 0.843 \\
\hline$I k b k b$ & -0.083 & 0.843 \\
\hline Sele & -0.083 & 0.843 \\
\hline Il17 & -0.083 & 0.843 \\
\hline Cd4Olg & -0.050 & 0.911 \\
\hline Myh6 & -0.033 & 0.948 \\
\hline$F n 1$ & -0.025 & 0.948 \\
\hline$I l 3$ & 0.000 & 1.018 \\
\hline Cd19 & 0.033 & 0.948 \\
\hline Csf1 & 0.041 & 0.911 \\
\hline Ccl2 & 0.050 & 0.911 \\
\hline Cxcr3 & 0.066 & 0.880 \\
\hline Cor4 & 0.116 & 0.776 \\
\hline$B c l 2 l 1$ & 0.150 & 0.708 \\
\hline$I l 12 a$ & 0.183 & 0.643 \\
\hline Csf3 & 0.183 & 0.643 \\
\hline Hladrb1 & 0.216 & 0.580 \\
\hline$I l 4$ & 0.233 & 0.551 \\
\hline Smad3 & 0.250 & 0.520 \\
\hline$I l 12 b$ & 0.250 & 0.520 \\
\hline$T g f b$ & 0.266 & 0.493 \\
\hline Il9 & 0.300 & 0.436 \\
\hline Il6 & 0.350 & 0.358 \\
\hline Lta & 0.400 & 0.291 \\
\hline Agtr1 & 0.433 & 0.249 \\
\hline Il18 & 0.466 & 0.212 \\
\hline Col4a5 & 0.483 & 0.193 \\
\hline Smad7 & 0.483 & 0.193 \\
\hline Cyp7a1 & 0.483 & 0.193 \\
\hline$B c l 2$ & 0.500 & 0.177 \\
\hline Edn1 & 0.533 & 0.147 \\
\hline Il15 & 0.566 & 1.206 \\
\hline Gusb & 0.633 & 0.076 \\
\hline $\mathrm{Cd} 34$ & 0.650 & 0.066 \\
\hline Ece1 & 0.666 & 0.058 \\
\hline Ski & 0.700 & 0.043 \\
\hline$C d 40$ & 0.700 & 0.043 \\
\hline Il7 & 0.733 & 0.031 \\
\hline Tnfrsf18 & 0.783 & 0.017 \\
\hline
\end{tabular}

Gene expression modulation was calculated as $\log _{2}$ fold change 4 weeks antiTNF therapy/baseline. Clinical response was calculated as percentage of PASI score decrease after 12 weeks of therapy. Correlation between gene expression modulation and clinical response to therapy was evaluated by Spearman's rank test (Spearman's $r$ ). Significance level was indicated ( $P$ value).

collected at baseline and after 4 weeks of treatment in the same lesional area. We first performed H\&E staining (Figure 3A) that showed compact lymphoid aggregates in the papillary dermis, immediately under the hyperproliferative stratum of the epidermis, at baseline. Images clearly evidence the disaggregation of the lymphoid-like tissue within the papillary dermis (red circles) after anti-TNF treatment.

To further characterize the lymphoid aggregates for the presence of $\mathrm{CCR} 7^{+}$cells, we next performed immunofluorescence 
A
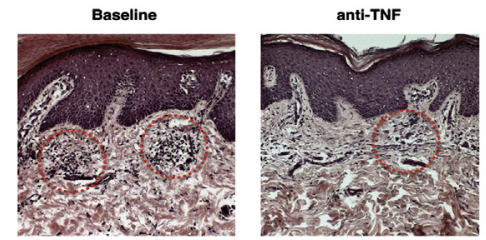

B
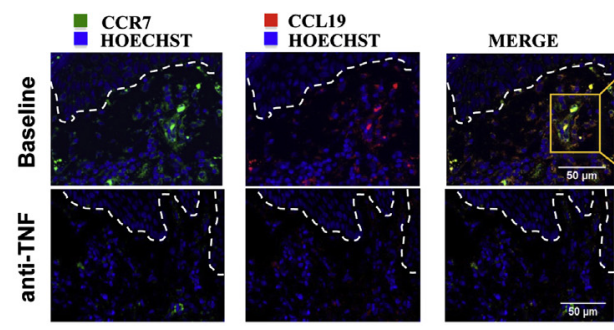

C
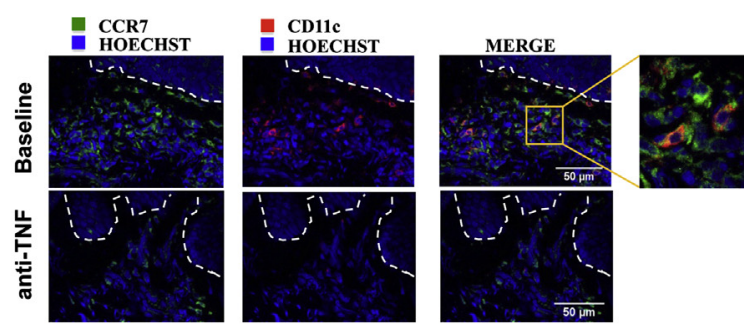

D

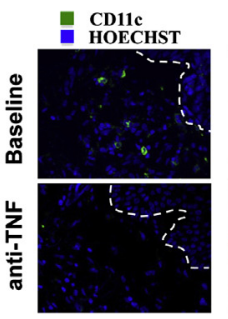

CD4
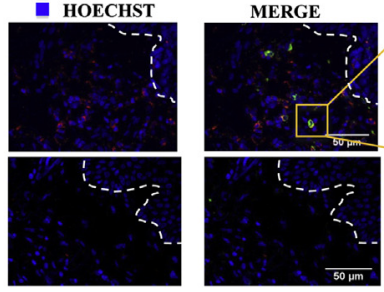

o
Figure 3 Four weeks of anti-TNF therapy induce lymphoid aggregate dissolution and reduction of $\mathrm{CCR}^{+}{ }^{+} \mathrm{CD} 11 \mathrm{C}^{+} \mathrm{DCs}$ and CCL19 in the subpapillary dermis. Punch skin biopsy specimens were taken from the same lesional area at baseline and after 4 weeks of anti-TNF treatment (etanercept) from individual responder patients. A: H\&E staining showing the lymphoid-like tissue within the papillary dermis (red circles). Immunofluorescence analysis was performed with anti-CCR7 (Alexa 488-green) and anti-CCL19 (Alexa 647-red) antibodies (B), anti-CCR7 (Alexa 488-green) and anti-CD11c (Alexa 647-red) antibodies (C), and anti-CD11c (Alexa 488green) and anti-CD4 (Alexa 647-red) antibodies (D). The figure shows one representative patient of three. Original magnification, $\times 10$, H\&E staining; $\times 40$, immunofluorescence images. White dotted lines, epidermal-dermal junctions.

microscopy on tissue sections collected at baseline and after 4 weeks of treatment.

Immunofluorescence analysis highlighted colocalization of $\mathrm{CCR}^{+}$cells with CCL19 to form lymphoid aggregates within the papillary dermis immediately below the hyperproliferative layers of the epidermis (Figure 3B). More important, treatment with TNF inhibitors strongly decreased $\mathrm{CCR} 7^{+}$cells in the upper dermis, in parallel with a decrease of CCL19 expression (Figure 3B). Consistently, quantification analysis showed that both CCR7 and CCL19 signals were significantly reduced after anti-TNF therapy $[42.11 \%$ of decrease $(P=0.0004)$ and $92.75 \%$ of decrease $(P<$ 0.0001), respectively] (Supplemental Figure S2).
To further characterize $\mathrm{CCR} 7^{+}$cells detected within the lymphoid aggregate, we next performed a double staining of tissue sections with anti-CCR7 and anti-CD11c antibodies. As shown in Figure 3C, most of the CD11 ${ }^{+}$cells expressed CCR7, and these cells were dramatically reduced after 4 weeks of anti-TNF treatment $(77.7 \%$ of decrease after therapy, $P=0.0023$ ) (Supplemental Figure S2). To confirm a direct effect of TNF inhibitors on DC maturation and on CCR7 expression, we performed in vitro experiments by culturing monocyte-derived DCs with TNF $\alpha / \mathrm{IL}-1 \beta$ in the presence or absence of etanercept. The results showed that CD83 and CCR7 expression, induced by TNF, was reduced in the presence of etanercept, thus underlining that TNF blocking agents could inhibit CCR7 expression by blocking DC maturation induced by inflammatory stimuli (Supplemental Figure S3). In addition, qPCR analysis performed on DCs in vitro also showed a marked induction of CCR7 and CCL19 expression by TNF $\alpha / \mathrm{IL}-1 \beta$ that was strongly inhibited by the addition of etanercept (Supplemental Figure S4). This definitely shows that TNF blockade directly interferes with the CCR7/CCL19 axis.

Notably, double staining with anti-CD11c and anti-CD4 antibodies clearly evidenced a physical interaction between $\mathrm{CD} 11 \mathrm{c}^{+} \mathrm{DCs}$ and $\mathrm{CD} 4^{+} \mathrm{T}$ cells within the lymphoid aggregate (Figure 3D). Four weeks of treatment with TNF inhibitors led to a strong reduction of $\mathrm{CD} 11 \mathrm{c}^{+}$cells and $\mathrm{CD} 4^{+} \mathrm{T}$ cells (87.2\% and $74.66 \%$, respectively; $P<0.0001$ ) (Figure 3D).

CCR7 was also expressed with CD4 on T cells within the lymphoid aggregate (Figure 4A and Supplemental Figure S5),

A
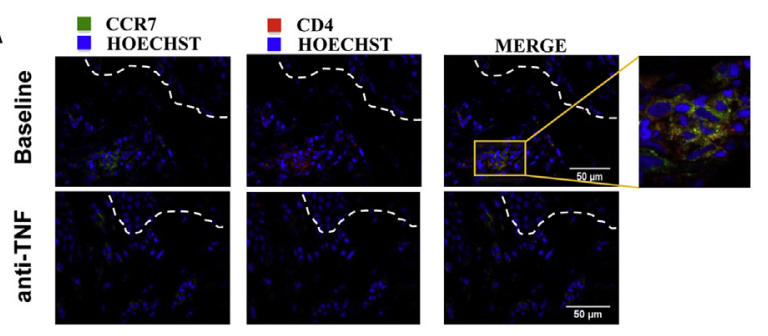

B
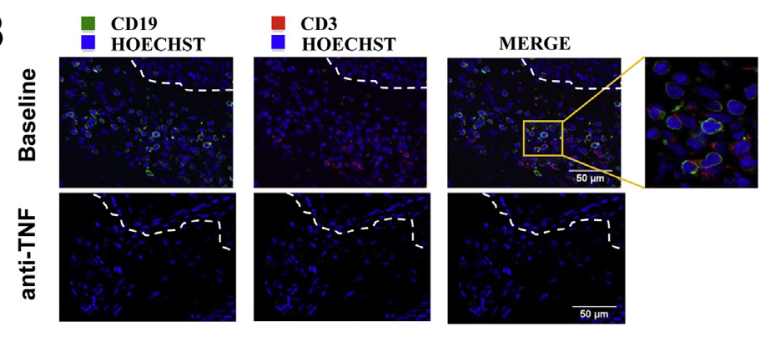

Figure 4 Lymphoid aggregates of the papillary dermis contain $\mathrm{CCR7}^{+} \mathrm{CD}^{+}$central memory/naïve $\mathrm{T}$ cells and $\mathrm{CD} 19^{+} \mathrm{B}$ cells. Punch skin biopsy specimens were taken from the same lesional area at baseline and after 4 weeks of anti-TNF therapy from individual responder patients; cryosections were prepared and stained for double-labeled immunofluorescence analysis with anti-CCR7 (Alexa 488-green) and anti-CD4 (Alexa 647-red) antibodies (A) and anti-CD19 (Alexa 488-green) and anti-CD3 (Alexa 647-red) antibodies (B). The figure shows one representative patient of three. Original magnification, $\times 40$. White dotted lines, epidermal-dermal junctions. 
whereas $\mathrm{CD} 8^{+} \mathrm{T}$ cells represented only a minor fraction of the $\mathrm{CCR} 7^{+}$cells (Supplemental Figure S5). In addition, we found that the lymphoid aggregate also includes $\mathrm{CD} 19^{+} \mathrm{B}$ cells (Figure 4B). However, their amount and decrease on therapy was not homogeneous among patients, in accordance with the lack of correlation with the clinical remission observed from gene expression data.

Together, these data clearly show that $\mathrm{CD} 11 \mathrm{c}^{+} / \mathrm{CCR} 7^{+}$ DCs and central memory/naive $\mathrm{CCR} 7^{+} / \mathrm{CD}^{+}{ }^{+} \mathrm{T}$ cells are components of the lymphoid aggregates in the papillary dermis and underline the role of their interaction in the generation of the proinflammatory network in the psoriatic plaque.

\section{Discussion}

The results of this study show that an early critical event for the resolution of psoriatic skin lesions mediated by TNF-blocking agents is the inhibition of the CCR7/CCL19 axis. Indeed, using a translational approach that correlates gene expression fold change in lesional skin with the PASI score decrease induced by TNF blockade, we found that, among genes that were modulated by the therapy, those that mostly associated with clinical remission were $\mathrm{Ccr} 7$, its ligand, $\mathrm{Ccl19}$, and genes involved in DC maturation (Cd80 and $C d 86$ ). In parallel, the results showed high correlation for T-cell activation genes (Il2ra, Icos, and Ctla4) and Vegf, encoding the VEGF.

The interaction between the chemokine, CCL19, and its receptor, CCR7, expressed by central memory/naïve $\mathrm{T}$ cells and maturing DCs, represents a key event for the recruitment of these cells to the lymph nodes. In psoriatic skin lesions, the presence of a lymphoid-like tissue has been observed in the dermis, and although this article was in preparation, a study by Mitsui et $\mathrm{al}^{20}$ evidenced the presence of lymphoid aggregates characterized by the expression of CCL19 and CCR7, suggesting a role for the CCR7/CCL19 axis in disease pathogenesis. Our study confirms the hypothesis of a role of CCR7/CCL19 interaction in the formation of dermal lymphoid-like tissue and provides new evidence for the role of the CCR7/CCL19 axis in disease pathogenesis by showing that disease resolution by antiTNF therapy is strongly associated with inhibition of the CCR7/CCL19 axis and dissolution of the lymphoid aggregates.

In particular, our data show that the inhibition of the CCR7/CCL19 axis is an early critical event in disease remission. Indeed, the down-regulation of $\mathrm{Ccr} 7$ expression observed at 4 weeks of anti-TNF therapy also correlates with the clinical remission occurring at later time points, indicating that the inhibition of the CCR7/CCL19 axis is an initiating event that evolves in disease resolution in response to TNF inhibitors. Consistently, in vitro analysis on DCs showed that the TNF-induced expression of CCR7/CCL19 mRNA is almost completely inhibited by the addition of etanercept.
Collectively, our data assign to TNF a central role in promoting the key interactions for the formation of dermal lymphoid aggregates in the psoriatic plaque. An intriguing hypothesis for the development of the psoriatic plaque, provided by Bowcock and Krueger, ${ }^{21}$ involves the establishment of a perivascular aggregate of $\mathrm{T}$ cells and mature DCs in the dermis, which may work as a secondary lymphoid tissue.

Among its multiple functions, TNF is known to be involved in the maintenance of the secondary lymphoid organ architecture and in promoting angiogenesis through the induction of VEGF. ${ }^{2-26}$

In human DCs, TNF has also been shown to induce the expression of $\mathrm{Ccr} 7 \mathrm{mRNA},{ }^{27}$ and herein we evidenced that it is capable of inducing detectable expression of CCR7 and of the maturation marker, CD83, in vitro. The addition of etanercept decreased both CD83 and CCR7 expression, thus showing that TNF inhibitors can interfere with DC maturation and CCR7 expression. Consistently, in vitro experiments on human DCs have demonstrated that TNF neutralization decreased CCL19 expression, induced by maturation stimuli (Supplemental Figure S4). ${ }^{28}$

A previous study has also reported a reduction of dermal DCs as the result of apoptosis in etanercept-treated patients responding to the therapy. ${ }^{29}$

Finally, our results also highlight that down-regulation of $V e g f$, together with decreased expression of $C c r 7$ and $C c l 19$, strongly correlated with disease resolution induced by TNF blockade, thus suggesting these three TNF targets as the main upstream components of the self-maintaining disease cycle.

From these results, TNF emerges as a pivotal player in the pathogenesis of psoriasis by promoting the formation of the lymphoid aggregates in the dermis for the generation and amplification of Th1 and Th17 responses.

After 4 weeks of treatment, we also found a detectable decrease of $I l 17$ expression, in accordance with a previous study, ${ }^{30}$ but we did not find a significant correlation with the PASI score decrease. In another study, it has been reported that the down-regulation of Th17 pathway genes at 12 weeks of etanercept treatment discriminates between responder and nonresponder patients. ${ }^{13}$ A possible explanation for this apparent discrepancy is that the Th17 response may represent a downstream effector arm that is generated and amplified by the pathogenic cycle maintained by CCR7/CCL19 interaction; therefore, its correlation with the clinical response is likely to become evident at later time points.

In conclusion, we found that early critical events for disease resolution, induced by TNF blockade, are the inhibition of the CCR7/CCL19 axis, paralleled with decreased DC maturation and VEGF down-regulation, which are associated with the breakup of the lymphoid aggregates in the upper dermis of psoriatic plaques. These findings strongly support the key role of the CCR7/CCL19 axis in the formation of the ectopic lymphoid aggregates in psoriatic skin and their role in disease pathogenesis. 


\section{Acknowledgments}

We thank Prof. Antonio Siccardi (IRCCS Ospedale San Raffaele, Milan, Italy) for helpful discussion and critical reading of the manuscript and Dr. Cesare Covino (Advanced Light and Electron Microscopy BioImaging Center, IRCCS Ospedale San Raffaele) for the support in fluorescence signal quantification.

\section{Supplemental Data}

Supplemental material for this article can be found at http://dx.doi.org/10.1016/j.ajpath.2013.04.021.

\section{References}

1. Lowes MA, Bowcock AM, Krueger JG: Pathogenesis and therapy of psoriasis. Nature 2007, 445:866-873

2. Nestle FO, Kaplan DH, Barker J: Psoriasis. N Engl J Med 2009, 361: 496-509

3. Yao Y, Richman L, Morehouse C, de los Reyes M, Higgs BW, Boutrin A, White B, Coyle A, Krueger J, Kiener PA, Jallal B: Type I interferon: potential therapeutic target for psoriasis? PLoS One 2008, 3:e2737

4. Zaba LC, Krueger JG, Lowes MA: Resident and "inflammatory" dendritic cells in human skin. J Invest Dermatol 2009, 129:302-308

5. Zhou X, Krueger JG, Kao MC, Lee E, Du F, Menter A, Wong WH, Bowcock AM: Novel mechanisms of T-cell and dendritic cell activation revealed by profiling of psoriasis on the 63,100-element oligonucleotide array. Physiol Genomics 2003, 13:69-78

6. Gottlieb AB, Chamian F, Masud S, Cardinale I, Abello MV, Lowes MA, Chen F, Magliocco M, Krueger JG: TNF inhibition rapidly down-regulates multiple proinflammatory pathways in psoriasis plaques. J Immunol 2005, 175:2721-2729

7. Zaba LC, Cardinale I, Gilleaudeau P, Sullivan-Whalen M, SuarezFarinas M, Fuentes-Duculan J, Novitskaya I, Khatcherian A, Bluth MJ, Lowes MA, Krueger JG: Amelioration of epidermal hyperplasia by TNF inhibition is associated with reduced Th17 responses. J Exp Med 2007, 204:3183-3194

8. Andreakos ET, Foxwell BM, Brennan FM, Maini RN, Feldmann M: Cytokines and anti-cytokine biologicals in autoimmunity: present and future. Cytokine Growth Factor Rev 2002, 13:299-313

9. Feldmann M, Maini RN: Anti-TNF alpha therapy of rheumatoid arthritis: what have we learned? Annu Rev Immunol 2001, 19:163-196

10. Katou F, Ohtani H, Nakayama T, Nagura H, Yoshie O, Motegi K: Differential expression of CCL19 by DC-Lamp+ mature dendritic cells in human lymph node versus chronically inflamed skin. J Pathol 2003, 199:98-106

11. Yarilina A, Park-Min KH, Antoniv T, Hu X, Ivashkiv LB: TNF activates an IRF1-dependent autocrine loop leading to sustained expression of chemokines and STAT1-dependent type I interferon-response genes. Nat Immunol 2008, 9:378-387

12. Bosè F, Raeli L, Garutti C, Frigerio E, Cozzi A, Crimi M, Caprioli F, Scavelli R, Altomare G, Geginat J, Abrignani S, Reali E: Dual role of anti-TNF therapy: enhancement of TCR-mediated $\mathrm{T}$ cell activation in peripheral blood and inhibition of inflammation in target tissues. Clin Immunol 2011, 139:164-176

13. Zaba LC, Suárez-Farinas M, Fuentes-Duculan J, Nograles KE, Guttman-Yassky E, Cardinale I, Lowes MA, Krueger JG: Effective treatment of psoriasis with etanercept is linked to suppression of IL-17 signaling, not immediate response TNF genes. J Allergy Clin Immunol 2009, 124:1022-1030.e395
14. Caprioli F, Bosè F, Rossi R, Petti L, Viganò C, Ciafardini C, Raeli L, Basilisco G, Ferrero S, Pagani M, Conte D, Altomare G, Monteleone G, Abrignani S, Reali E: Reduction of CD68+ macrophages and decreased IL-17 expression in intestinal mucosa of patients with inflammatory bowel disease strongly correlate with endoscopic response and mucosal healing following infliximab therapy. Inflamm Bowel Dis 2013, 19:729-739

15. Taylor PC, Peters AM, Paleolog E, Chapman PT, Elliott MJ, McCloskey R, Feldmann M, Maini RN: Reduction of chemokine levels and leukocyte traffic to joints by tumor necrosis factor alpha blockade in patients with rheumatoid arthritis. Arthritis Rheum 2000, 43:38-47

16. Brown D, Lydon J, McLaughlin M, Stuart-Tilley A, Tyszkowski R, Alper S: Antigen retrieval in cryostat tissue sections and cultured cells by treatment with sodium dodecyl sulfate (SDS). Histochem Cell Biol 1996, 105:261-267

17. Li P, Liu P, Xiong RP, Chen XY, Zhao Y, Lu WP, Liu X, Ning YL, Yang N, Zhou YG: Ski, a modulator of wound healing and scar formation in the rat skin and rabbit ear. J Pathol 2011, 223: 659-671

18. Banchereau J, Briere F, Caux C, Davoust J, Lebecque S, Liu YJ, Pulendran B, Palucka K: Immunobiology of dendritic cells. Annu Rev Immunol 2000, 18:767-811

19. van Kooten C, Banchereau J: Functions of CD40 on B cells, dendritic cells and other cells. Curr Opin Immunol 1997, 9:330-337

20. Mitsui H, Suarez-Farinas M, Belkin DA, Levenkova N, FuentesDuculan J, Coats I, Fujita H, Krueger JG: Combined use of laser capture microdissection and cDNA microarray analysis identifies locally expressed disease-related genes in focal regions of psoriasis vulgaris skin lesions. J Invest Dermatol 2012, 132:1645-1655

21. Bowcock AM, Krueger JG: Getting under the skin: the immunogenetics of psoriasis. Nat Rev Immunol 2005, 5:826

22. Aloisi F, Pujol-Borrell R: Lymphoid neogenesis in chronic inflammatory diseases. Nat Rev Immunol 2006, 6:205-217

23. Pasparakis M, Alexopoulou L, Episkopou V, Kollias G: Immune and inflammatory responses in TNF alpha-deficient mice: a critical requirement for TNF alpha in the formation of primary B cell follicles, follicular dendritic cell networks and germinal centers, and in the maturation of the humoral immune response. J Exp Med 1996, 184: 1397-1411

24. Randall TD, Carragher DM, Rangel-Moreno J: Development of secondary lymphoid organs. Annu Rev Immunol 2008, 26:627-650

25. Heidenreich R, Rocken M, Ghoreschi K: Angiogenesis drives psoriasis pathogenesis. Int J Exp Pathol 2009, 90:232-248

26. Lew W, Bowcock AM, Krueger JG: Psoriasis vulgaris: cutaneous lymphoid tissue supports T-cell activation and "Type 1" inflammatory gene expression. Trends Immunol 2004, 25:295-305

27. Geissmann F, Dieu-Nosjean MC, Dezutter C, Valladeau J, Kayal S, Leborgne M, Brousse N, Saeland S, Davoust J: Accumulation of immature Langerhans cells in human lymph nodes draining chronically inflamed skin. J Exp Med 2002, 196:417-430

28. van Lieshout AW, Barrera P, Smeets RL, Pesman GJ, van Riel PL, van den Berg WB, Radstake TR: Inhibition of TNF alpha during maturation of dendritic cells results in the development of semi-mature cells: a potential mechanism for the beneficial effects of TNF alpha blockade in rheumatoid arthritis. Ann Rheum Dis 2005, 64:408-414

29. Malaviya R, Sun Y, Tan JK, Wang A, Magliocco M, Yao M, Krueger JG, Gottlieb AB: Etanercept induces apoptosis of dermal dendritic cells in psoriatic plaques of responding patients. J Am Acad Dermatol 2006, 55:590-597

30. Wang F, Smith N, Maier L, Xia W, Hammerberg C, Chubb H, Chen C, Riblett M, Johnston A, Gudjonsson JE, Helfrich Y, Kang S, Fisher GJ, Voorhees JJ: Etanercept suppresses regenerative hyperplasia in psoriasis by acutely downregulating epidermal expression of interleukin (IL)-19, IL-20 and IL-24. Br J Dermatol 2012, 167: 92-102 